\title{
Viscodelamination at the vitreoretinal juncture in severe diabetic eye disease
}

\author{
DAVID MCLEOD AND CHARLES R JAMES \\ From the Surgical Vitreoretinal Unit, Moorfields Eye Hospital, London
}

SUMmaRY Injection of $1 \%$ methylcellulose or $1 \%$ sodium hyaluronate (Healonid) was used to separate attached vitreous cortex and fibrovascular epiretinal membranes from the retina in 40 eyes undergoing closed microsurgery for severe diabetic eye disease. The viscodelamination technique was of great value in elevating vitreous cortex or sparsely vascularised epiretinal membranes, especially in eyes with combined traction and rhegmatogenous retinal detachment. However, bleeding from or tearing of the retina limited the usefulness of this technique in the surgery of highly vascularised and adherent membranes, as in eyes with table-top traction retinal detachment. Recurrent epiretinal membrane proliferation was seen in some eyes postoperatively.

Proliferative diabetic retinopathy (PDR) is characterised by outgrowth of new blood vessels and fibroblasts into the scaffold provided by attached cortical vitreous gel. The blinding sequelae of PDR - namely, retinal detachment and vitreous haemorrhage-result either from contraction of such fibrovascular epiretinal membranes (ERMs) or from avulsion of the edges of the membranes during posterior vitreous detachment (PVD) with retinal break formation or rupture of new vessels. The twin strategies underlying the surgical treatment of PDR complications are, firstly, reduction in the stimulus to vasoproliferation (for example, by means of scatter endophotocoagulation of ischaemic retina'), and secondly removal of postbasal vitreous cortex together with any fibrovascular tissue contained therein. ${ }^{2}$ Technical difficulties may arise, however, if the fibrovascular ERMs are excessively adherent or if the vitreous cortex remains extensively attached to the retina. ${ }^{3+}$ Stenkula and Tornquist described methods of elevating ERMs from the retina using sodium hyaluronate.

The purpose of this paper is to report our experience of using viscous fluids to separate cortical vitreous and ERMs from the retinal surface ('viscodelamination') during closed microsurgery for severe diabetic eye disease.

Correspondence to Mr D McLeod, FRCS, Moorfields Eye Hospital, City Road, London EC1V 2PD.

\section{Material and methods}

Between 1984 and 1986 a viscodelamination technique was employed during closed microsurgery for complications of PDR in 40 eyes of 38 patients in the Surgical Vitreoretinal Unit at Moorfields Eye Hospital, London. In the majority of procedures ( 28 eyes) $1 \%$ methylcellulose (formulated in Moorfields' pharmacy) was used; methylcellulose at $2 \%$ concentration (as used in anterior segment surgery) had been found to be excessively difficult to aspirate from the eye after viscodelamination. In the remaining 12 eyes sodium hyaluronate $1 \%$ (Healonid) was used.

Eyes suitable for viscodelamination were usually identified during preoperative ophthalmoscopic or ultrasonic examination by paying careful attention to the extent of PVD. Eyes with macular traction retinal detachment (10 eyes) or combined traction and rhegmatogenous detachment (18 eyes) but with minimal or limited $P V D$ were specially selected for viscodelamination (Fig. 1). In a further seven eyes viscodelamination was employed primarily to aid scissors dissection (segmentation or delamination) of widespread fibrovascular ERMs causing 'table-top' traction retinal detachment. It was relatively unusual for extensive non-separation of the vitreous cortex to accompany vitreous haemorrhage without significant retinal detachment (five eyes).

Healonid $1 \%$ (prepacked in a $2 \mathrm{ml}$ syringe) or $1 \%$ 


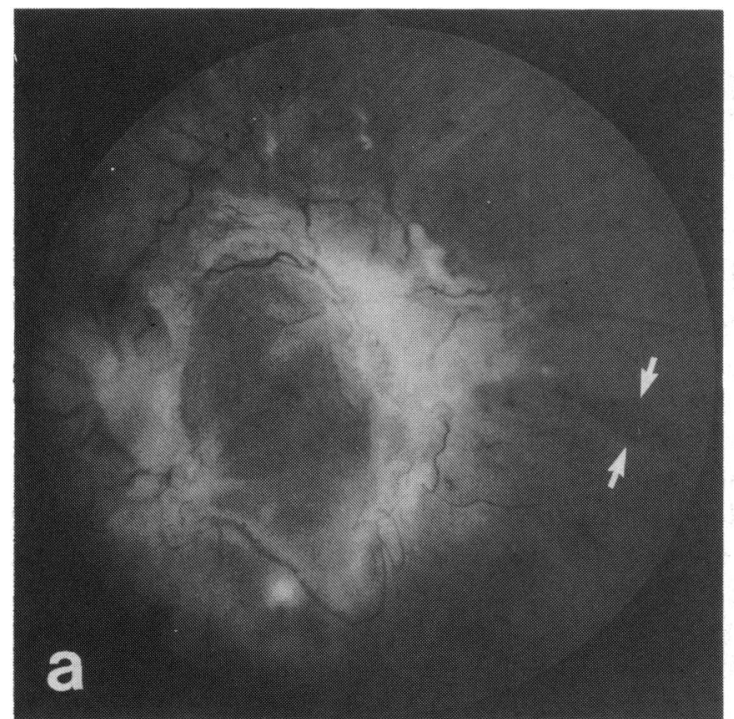

Fig. 1A

Fig. 1 Two eyes with combined traction and rhegmatogenous retinal detachment treated by vitrectomy, viscodelamination, and gas tamponade. (A) Preoperative fundus photograph with retinal break arrowed; acuity $=3 / 60$. (B) Same eye as (A) one month postoperatively; acuity $=6 / 12$. (C) Preoperative fundus photograph (note macular hole); acuity = hand motion. (D) Same eye as (C) two months postoperatively; acuity $=4 / 60$.

methylcellulose in a $5 \mathrm{ml}$ syringe was connected via flexible tubing to a 20 gauge cannula with a bent tip ('hockey stick'). After central vitrectomy by the

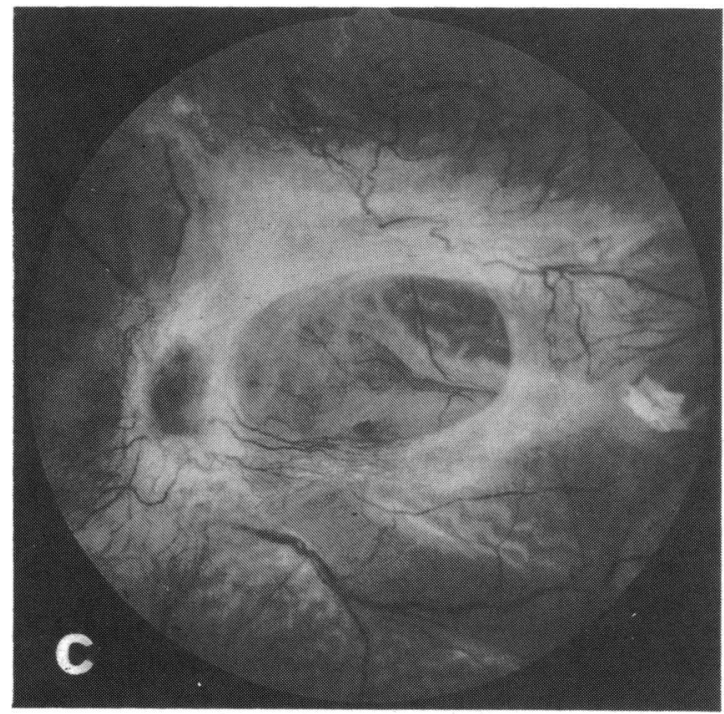

Fig. 1C

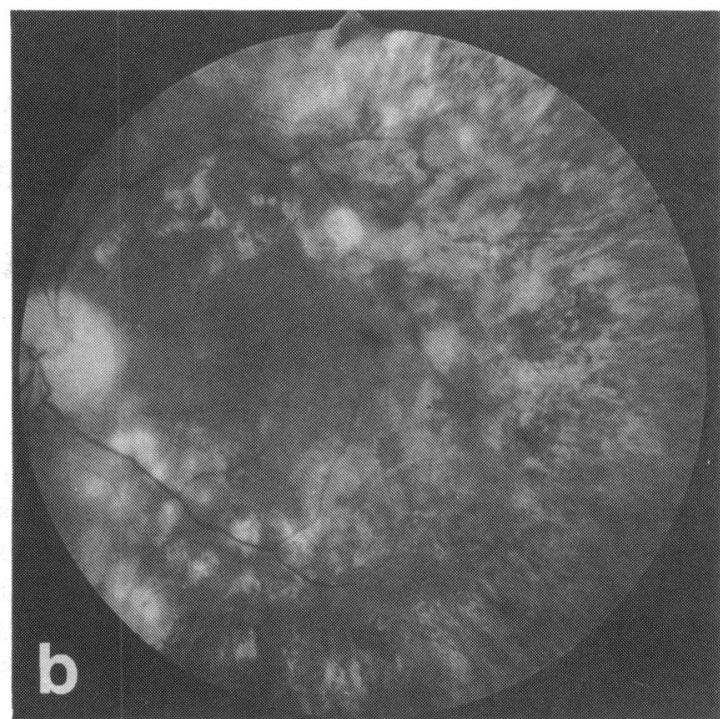

Fig. 1B

standard three-port system popularised by Charles,? $1-3 \mathrm{ml}$ of viscous fluid was injected between attached cortical vitreous gel and the retina via a pre-existing hiatus in an area of detached vitreous cortex or following creation of a $1 \mathrm{~mm}$ diameter hole in attached or detached cortex by means of a microvitreoretinal blade or the suction cutter (Fig. 2). The infusion fluid which was thus displaced anteriorly sometimes escaped from the eye round the fibreoptic endoilluminating probe or injection cannula; alternatively, fluid returned up the infusion cannula and

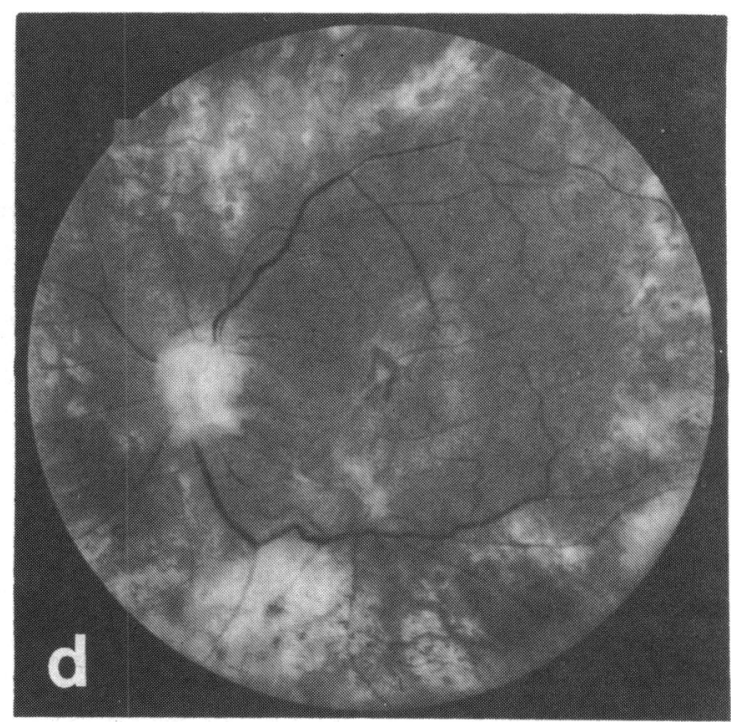

Fig. 1D 

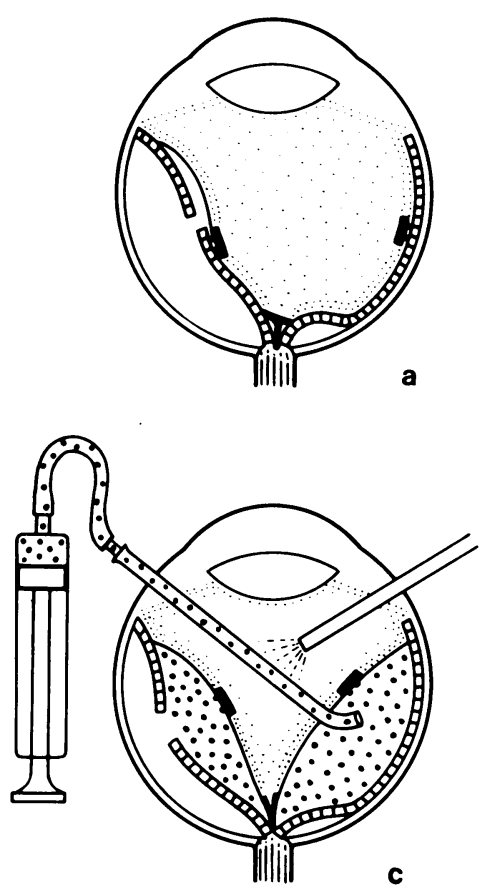
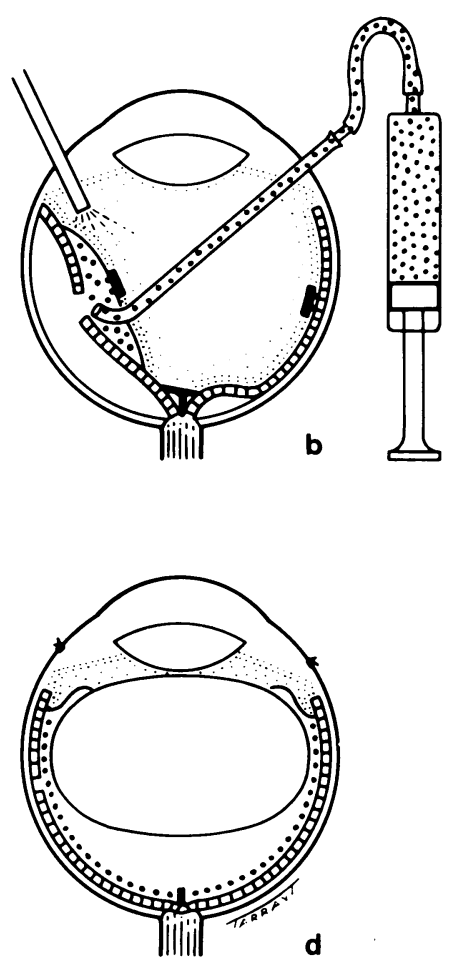

Fig. 2 Diagrams of the viscodelamination technique. (a) Combined traction and rhegmatogenous retinal detachment with vitreous cortex extensively attached. (b) Outset of viscous injection after central vitrectomy. (c) Completion of en bloc viscodelamination except at optic disc. (d) Internal tear closure by gas after completion of vitrectomy and aspiration of viscous injectate. tubing or was intermittently released from one of the two superior sclerotomies in response to ocular hypertension. The speed and volume of injection of viscous material was controlled by verbal instructions to the surgical assistant, who held the syringe in one hand while keeping the fundus-viewing infusion contact lens in suitable position on the cornea with his other hand.

Viscodelamination was accompanied by limited blunt mechanical separation of vitreous cortex with the curved cannula. In some eyes further holes were made in the vitreous cortex to permit complete postbasal separation of the vitreous through $360^{\circ}$, while traction on persisting adhesions between the retina and the ERMs was minimised. Local circumcision of vitreoretinal adhesions or scissors segmentation or delamination of membranes was carried out during, and/or at the completion of, the retrohyaloid viscous injection, while the cortical vitreous 'cone' remained virtually intact.

With the suction cutter vitrectomy was then completed (including removal of ERMs within the viscoelevated posterior vitreous cortex), and most of the retrohyaloid viscous injectate was aspirated. However, bipolar diathermy of bleeding points often revealed a persistent viscous layer on the retinal surface as indicated by temporary opacification during diathermy. Thereafter further measures to stabilise the posterior segment included internal drainage of subretinal fluid, fluid/gas or fluid/silicone oil exchange (Figs. 3, 4) and focal or scatter endophotocoagulation. The viscous fluid was not used primarily to achieve internal closure of retinal breaks. In view of the potential risk of reparative epiretinal fibrosis it was considered especially important to remove all viscous fluid from eyes into which silicone oil was subsequently injected (16 eyes).

The outcome of surgery was evaluated at six months postoperatively in all cases. Complete retinal reattachment or only localised, stable, extramacular traction retinal detachment was regarded as a successful anatomical outcome.

\section{Results}

In all but four eyes complete separation of nonvascularised postbasal vitreous cortex was achieved, so that after vitrectomy no connection persisted between any residual epiretinal fibrovascular tissue and the vitreous base. Indeed in most eyes no fibrovascular tissue remained except at the optic disc (Figs. 1-4). On only one occasion was injected viscous material identified in the subretinal space; otherwise the progressive separation of vitreous cortex and retina was associated with apparent flat- 


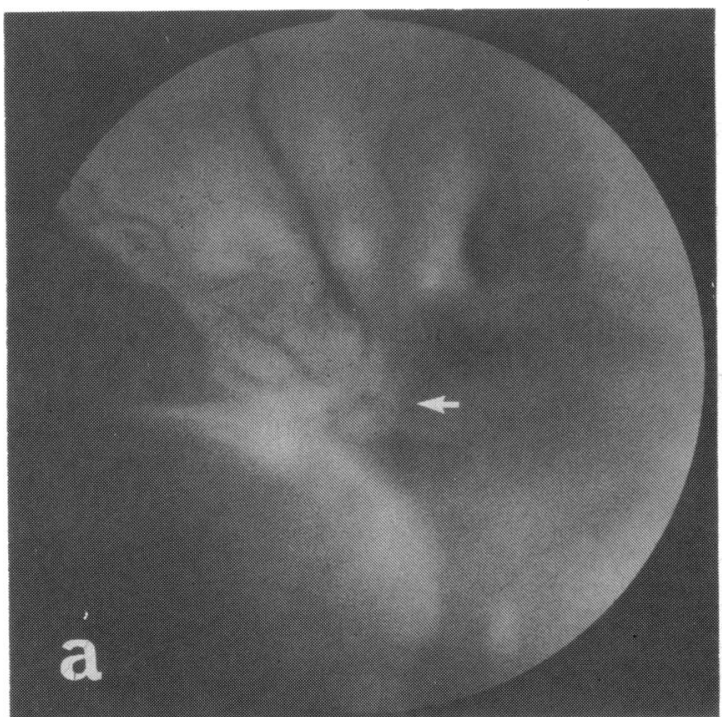

Fig. 3A

Fig. 3 Active proliferative retinopathy treated by vitrectomy, viscodelamination, endolaser, and silicone oil exchange. (A) Preoperative fundus photograph showing oedematous detached retina above optic disc (arrow) and dense premacular retrohyaloid haemorrhage; acuity =hand motion. (B) Same eye as (A) two days postoperatively.

(C) Same eye nine months later and following removal of silicone oil; acuity $=6 / 18$.

tening of the retina. However, preretinal haemorrhage was frequent during viscodelamination owing to avulsion of the vascularised adhesions between the

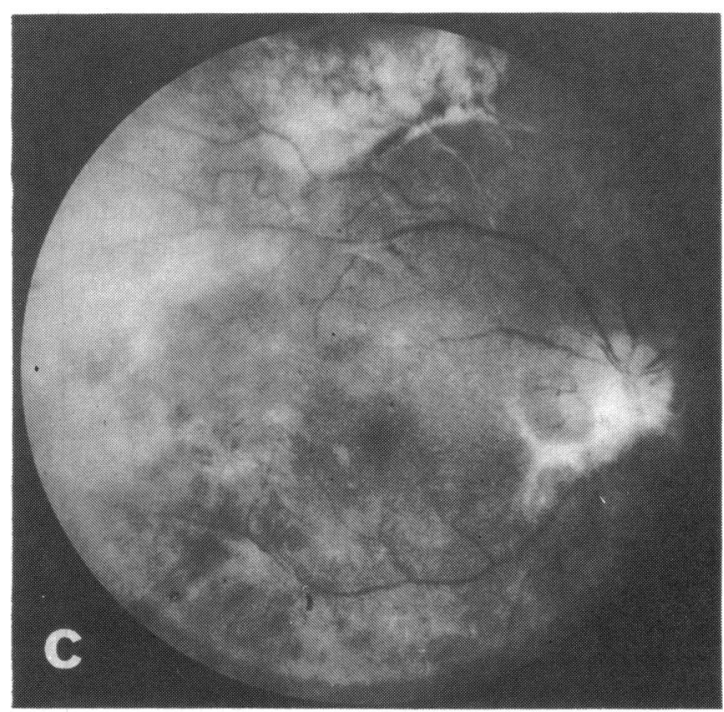

Fig. 3C

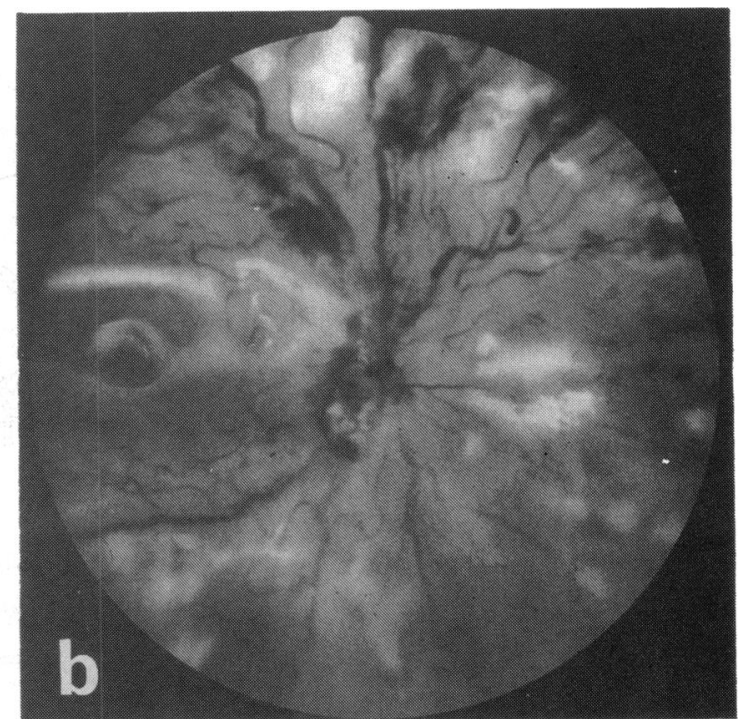

Fig. 3B

ERMs and the retina. Nevertheless the bleeding generally remained localised, and no difference was noted between Healonid $1 \%$ and methylcellulose $1 \%$ in respect of this intraoperative haemostasis or 'haemorrhage confinement'. Retinal tearing attributable to the viscodelamination technique was identified in 12 eyes (Table 1), while a retinal break near a surgical entry site (and possibly attributable to vitreous base herniation during the viscous injection) was found in four eyes at the end of the procedure.

No ocular hypertension or undue inflammation attributable to residual viscous injectate was evident postoperatively. A successful anatomical outcome (accompanied by visual improvement) was achieved in 28 of the 40 eyes (Table 1). Surgical failure reflected the development of macular distortion from reparative epiretinal fibrosis in two eyes, rubeotic glaucoma plus retro-silicone oil neovascularisation in one eye (Fig. 4), and combined traction and rhegmatogenous retinal redetachment in nine eyes.

Table 1 Results of viscodelamination in 40 eyes with PDR

\begin{tabular}{lccc}
\hline & $\begin{array}{l}\text { Number } \\
\text { ofeyes }\end{array}$ & $\begin{array}{l}\text { Successful } \\
\text { outcome }\end{array}$ & $\begin{array}{l}\text { VDL-induced } \\
\text { retinal breaks }\end{array}$ \\
\hline VH & 5 & 5 & 1 \\
TRD & 10 & 8 & 3 \\
CTRD & 18 & 11 & 4 \\
TTTRD & 7 & 4 & 4 \\
Totals & 40 & 28 & 12
\end{tabular}

VH = vitreous haemorrhage. $T R D=$ traction retinal detachment . CTRD $=$ combined traction and rhegmatogenous detachment TTTRD=table-top traction retinal detachment. $\mathrm{VDL}=$ viscodelamination 


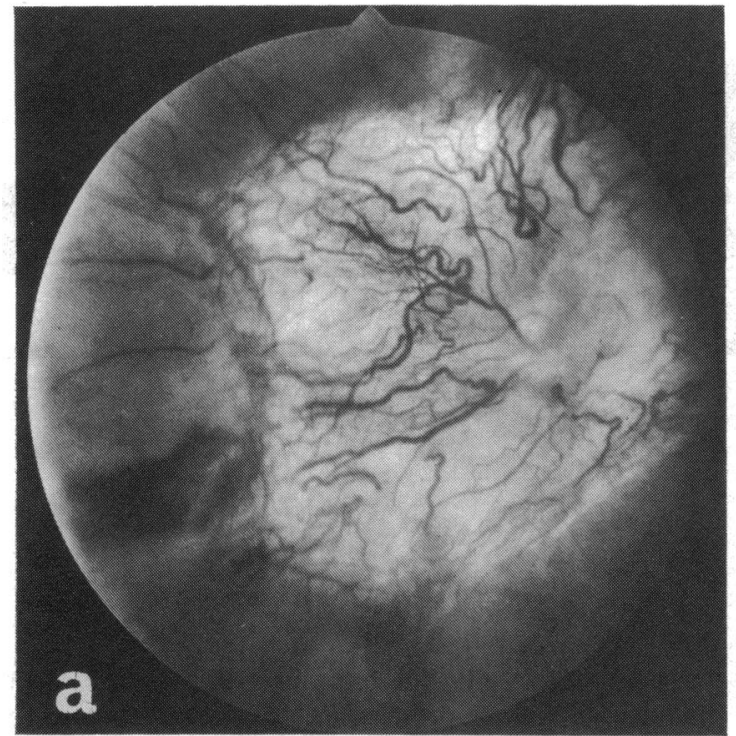

Fig. 4A

Fig. 4 Table-top traction retinal detachment treated by vitrectomy, viscodelamination, and silicone oil exchange. (A) Preoperative fundus photograph; acuity = hand motion. (B) Same eye as (A) three days postoperatively (single retinal break arrowed). (C) Retro-silicone oil neovascularisation (arrow) one month later.

In four of these nine eyes the presence of intravitreal silicone oil permitted 'rhegmatogenous confinement' of the detachment to an extramacular location, and all four had improved vision six months postoperatively.

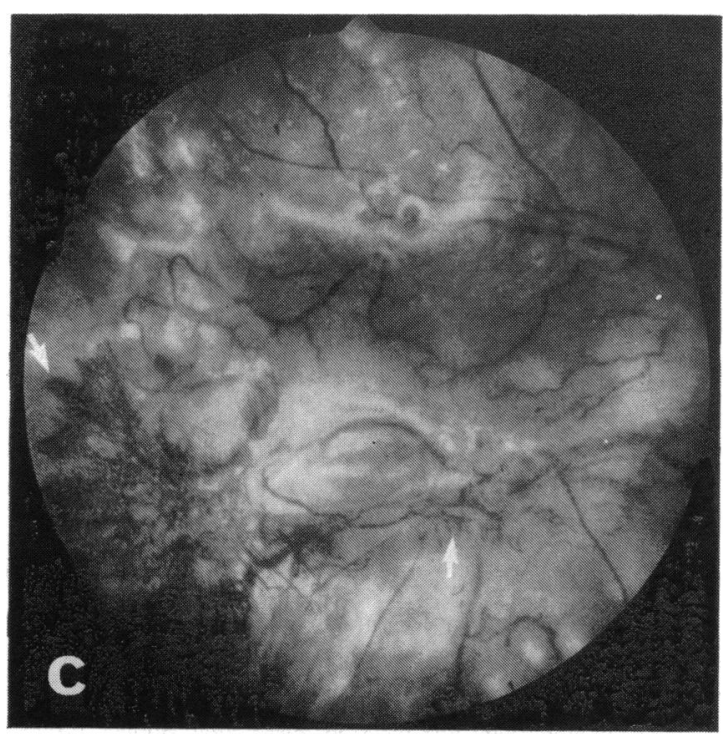

Fig. 4C

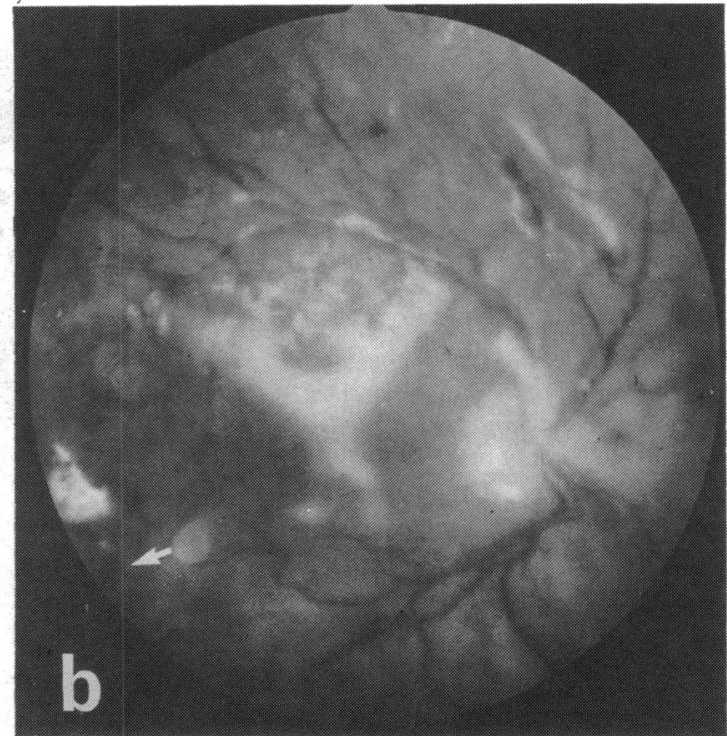

Fig. 4B

\section{Discussion}

Isolation of postbasal fibrovascular foci from the vitreous base by vitrectomy-circumcision reduces the tendency to postoperative rebleeding or retinal redetachment in PDR, ${ }^{8}$ while removal of all postbasal vitreous cortex usually prevents any further epiretinal neovascularisation. ${ }^{2}$ Even if the vitreous cortex remains attached to the retina preoperatively, these fundamental objectives of diabetic vitrectomy can often be achieved using microscissors, microforceps, etc. Such mechanical vitreoretinal separation may be facilitated by cortical gel condensation or fibroglial invasion of the vitreous cortex, often accompanied by multiple focal areas of PVD. ${ }^{9}$ Nevertheless viscodelamination of vitreous cortex from the retina often permits more rapid accomplishment of these objectives and is especially useful for separating multiple, sparsely vascularised ERMs in the midperiphery of phakic eyes. It has proved most valuable in eyes with combined traction and rhegmatogenous retinal detachment (in which separation of extensive acellular cortical vitreous from detached, ischaemic, and atrophic retina would otherwise have presented technical difficulties-Figs. 1, 2), and also in eyes with dense premacular retrohyaloid haemorrhage $\mathrm{e}^{1011}$ (Fig. 3). However, the viscodelamination technique carries with it a significant risk of retinal breaks, which are especially likely to arise when attempts are made to elevate extremely adherent fibrovascular ERMs. Recourse to scissors delamination is thus essential if viscous membranoretinal separation does not readily occur.

Intraoperative haemorrhage and fibrin clot forma- 
Fig. 5 Diagrams comparing viscodelamination (left) and scissors delamination (right) of fibrovascular epiretinal membrane; note avulsion of vascularised outgrowth and bleeding from side puncture of retinal vein during viscodelamination, and transection of vascularised outgrowth by scissors delamination.
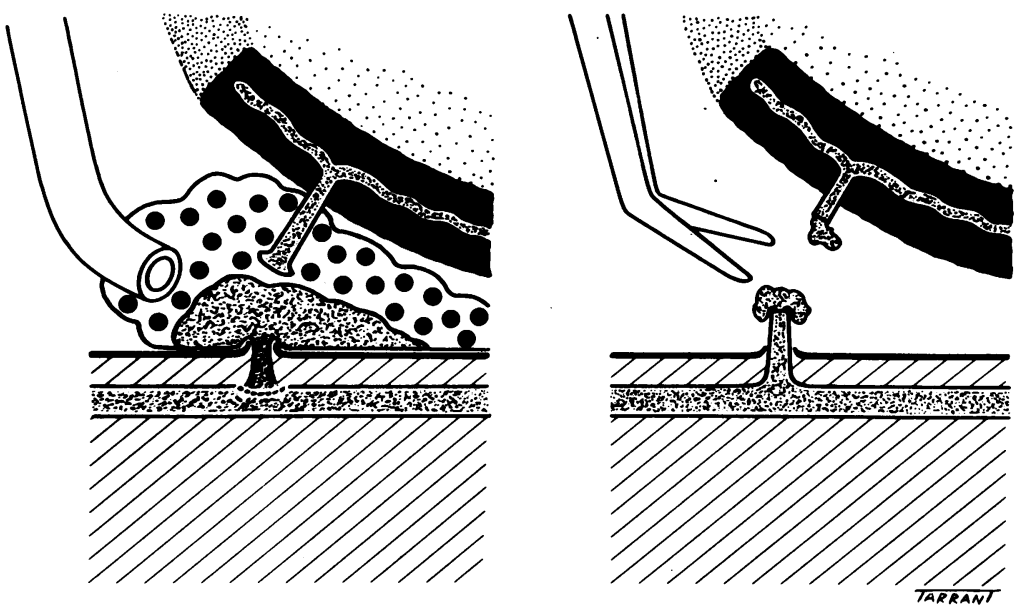

tion is a serious risk in all eyes with highly vascularised epiretinal membranes whatever technique is employed. In our experience, however, bleeding is much more problematic if a vascularised outgrowth is avulsed from the intraretinal circulationfor example, during membrane peeling, blunt mechanical delamination, or viscodelamination-in contrast to its preretinal division during scissors delamination. ' In the former case a 'side puncture' of a retinal vein is often slow to thrombose (Fig. 5), and haemostasis by diathermy necessitates retinal coagulation and retinal vascular occlusion. Scissors delamination of highly vascularised ERMs is a more appropriate technique, since the retinal cut end of the outgrowth seldom bleeds profusely (Fig. 5), or it can be selectively diathermised with minimal damage to the underlying retina. For those reasons we have now virtually abandoned the use of viscodelamination for removal of highly vascularised ERMs-for example, in table-top traction retinal detachment.

At the outset of this study we had expected that the relatively atraumatic interfacial separation of vitreous and retina by means of a viscous injection would prevent recurrence of ERMs postoperatively. ${ }^{612}$ However, even in eyes where vitreoretinal separation was achieved entirely by viscodelamination (unaccompanied by retinal break formation, haemorrhage, or recourse to silicone oil injection) reparative epiretinal fibrosis sometimes developed. Notwithstanding a possible role of residual preretinal viscous fluid in this process, but as is also the case after epimacular membrane peeling, ${ }^{1314}$ membrane recurrence appears to be related to the underlying disease,$^{15}$ in this case ischaemic diabetic retinopathy. Moreover, when such an eye contains silicone oil, recurrent epiretinal neovascularisation may occur behind the oil globule despite complete prior removal of the cortical vitreous scaffold by viscodelamination
(Fig. 4). This remarkable phenomenon may reflect increased retinal production of vasoproliferative factors in some silicone oil filled diabetic eyes because of reduced oxygen diffusion from the vitreous cavity into ischaemic areas of retina (Landers R, personal communication, 1986). Such diffusion otherwise appears to limit the damage resulting from retinal vascular non-perfusion. ${ }^{1617}$ Thus the use of viscous fluids in diabetic vitrectomy, whether an aqueous solution or silicone oil,,$^{12}$ gives occasion for both tangible benefits and significant complications.

We are indebted to Miss Heather Lucas for secretarial assistance, to Mr K Sehmi for the clinical photographs, and to Mr T Tarrant for the diagrams.

\section{References}

1 Ficker LA, Passani F, Leaver PK, McLeod D. Xenon-arc endophotocoagulation during vitrectomy for diabetic vitreous haemorrhage. Graefs Arch Clin Exp Ophthalmol 1986; 224: 423-7.

2 Michels RG. Vitrectomy for complications of diabetic retinopathy. Arch Ophthalmol 1978; 96: 237-46.

3 Blankenship GW. Preoperative prognostic factors in diabetic pars plana vitrectomy. Ophthalmology 1982; 89: 1246-9.

4 Barrie T, Feretis EB, Leaver PK, McLeod D. Closed microsurgery for diabetic traction macular detachment. $\mathrm{Br} J$ Ophthalmol 1982; 66: 754-8.

5 Stenkula $S$, Tornquist $R$. Use of Healon in vitrectomy and difficult retinal detachments. In: Miller D, Stegman R, cds. Healon (sodium hyaluronate): a guide to its use in ophthalmic surgery. New York: Wiley, 1983: 207-21.

6 Barry PJ, Hiscott PS, Grierson I, Marshall J, McLeod D. Reparative epiretinal fibrosis after diabetic vitrectomy. Trans Ophthalmol Soc UK 1985; 104: 285-96.

7 Charles S. Vitreous microsurgery. Baltimore, London: Williams and Wilkins, 1981.

8 Irvine AR. Indications for vitrectomy via the pars plana. Trans Ophthalmol Soc UK 1975; 95: 436-43.

9 Faulborn J, Bowald S. Microproliferations in proliferative diabetic retinopathy and their relationship to the vitrcous: corresponding light and electron microscopic studics. Graefes Arch Clin Exp Ophthalmol 1985; 223: 130-8. 
10 O'Hanley GP, Canny CLB. Diabetic dense premacular haemorrhage: a possible indication for prompt vitrectomy. Ophthalmology 1985; 92: 507-11.

11 Ramsay RC, Knobloch WH, Cantrill HL. Timing of vitrectomy for active proliferative diabetic retinopathy. Ophthalmology 1986; $93: 283-8$.

$12 \mathrm{McLeod} \mathrm{D}$. Silicone-oil injection during closed microsurgery for diabetic retinal detachment. Graefes Arch Clin Exp Ophthalmol 1986; 224: 55-9.

13 Hiscott PS, Grierson I, McLeod D. Natural history of fibrocellular epiretinal membranes: a quantitative, autoradiographic, and immunohistochemical study. Br J Ophthalmol 1985; 69: 810-23.
14 Margherio RR, Cox MS, Trese MT, Murphy PL, Johnson J, Minor LA. Removal of epimacular membranes. Ophthalmology 1985; 92: 1075-82.

15 McLeod D, Hiscott PS, Grierson I. Age-related cellular proliferation at the vitreoretinal juncture. Eye 1987; 1: 263-81.

16 McLeod D. Retinal ischaemia, disc swelling and axoplasmic transport. Trans Ophthalmol Soc UK 1976; 96: 313-6.

17 McLeod D, Marshall J, Kohner EM, Bird AC. The role of axoplasmic transport in the pathogenesis of retinal cotton-wool spots. Br J Ophthalmol 1977; 61: 177-91.

Accepted for publication 15 April 1987. 